This item was submitted to Loughborough's Research Repository by the author.

Items in Figshare are protected by copyright, with all rights reserved, unless otherwise indicated.

\title{
The simulation, modelling and sizing of pressure filters
}

PLEASE CITE THE PUBLISHED VERSION

PUBLISHER

(C) Elsevier

VERSION

AM (Accepted Manuscript)

LICENCE

CC BY-NC-ND 4.0

\section{REPOSITORY RECORD}

Tarleton, E.S., and Richard J. Wakeman. 2009. "The Simulation, Modelling and Sizing of Pressure Filters". figshare. https://hdl.handle.net/2134/4851. 
This item was submitted to Loughborough's Institutional Repository (https://dspace.lboro.ac.uk/) by the author and is made available under the following Creative Commons Licence conditions.

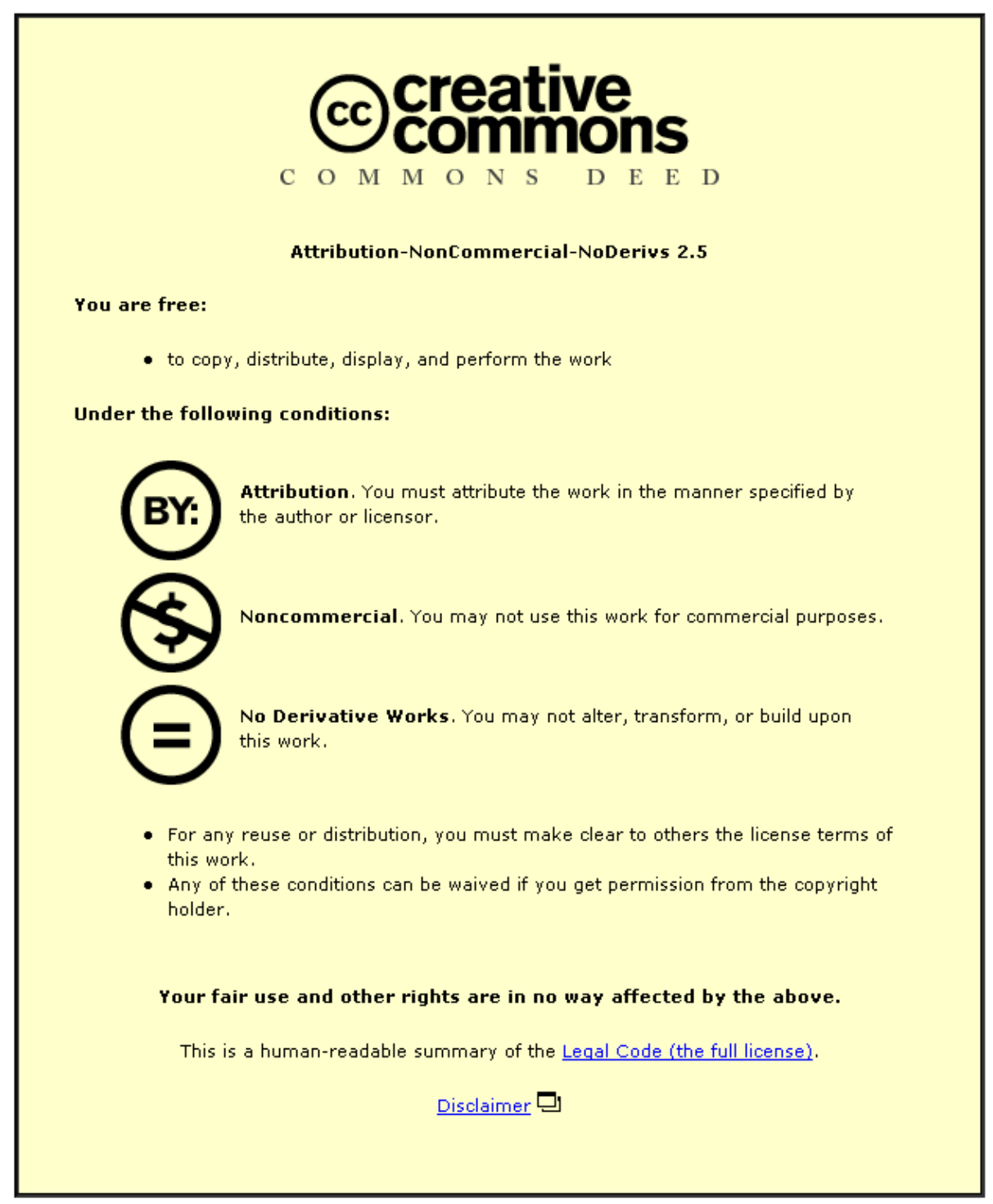

For the full text of this licence, please go to: http://creativecommons.org/licenses/by-nc-nd/2.5/ 


\title{
THE SIMULATION, MODELLING AND SIZING OF PRESSURE FILTERS
}

\author{
E.S. Tarleton ${ }^{\dagger}$ (e.s.tarleton@lboro.ac.uk) and R.J. Wakeman ${ }^{\dagger \dagger}$ \\ ${ }^{\dagger}$ Department Chemical Engineering, Loughborough University, Loughborough, Leics., UK. \\ ${ }^{\dagger+}$ Separation Processes Centre, University of Exeter, Devon, UK.
}

\section{ABSTRACT}

Some preliminary results from a research program aimed at providing computer simulations for solid/liquid separation processes are presented. The personal computer software package described models the operation of pressure filters. The example of the diaphragm filter press is chosen and it is shown how the integration of industrially proven research data with powerful modular software routines can simulate the cake formation, compression, dewatering and washing operations. With a knowledge of the intended operating conditions, the software generates extensive data for the filter cycle and minimises the time and cost associated with preliminary and pilot scale test work.

\section{INTRODUCTION}

Of the many unit operations available to the process engineer, those associated with solid/liquid separation are perhaps the most difficult to specify. For these systems it would be rare practice to use fundamental theoretical relationships for either the selection, design or optimisation of equipment with the result that the specification of chemical plant is rarely performed without recourse to extensive laboratory and pilot scale tests. Whilst the task of selecting a solid/liquid separator for a given duty has been considerably simplified by the recent introduction of the computer software $\mathrm{p}^{\mathrm{C}}$-SELECT ${ }^{1,2}$, its presence in the market place should be regarded in the context of an initial step. When a particular type of separator has been identified, the separations technologist is still faced with the prospect of undertaking an extensive test programme to enable accurate scale-up and the optimisation of size and configuration. These problems are compounded by the dearth of literature relating to such matters and the apparent lack of 'standard' procedures.

The authors have believed for many years that solid/liquid separation technology, whether it be in the areas of selection, design or optimisation, is best dealt with by interactive computer software. To be most effective the software must incorporate a well chosen mix of algorithms, expert system and input information from the user, be based on sound ideas and proven data from experimental and theoretical research projects and maintain the ability to give advice and education in a congenial fashion so that any psychological barriers to computer use are avoided.

This paper describes some preliminary results from a software package designed to simulate the operation of pressure filters. The software incorporates industrially proven methodologies which are combined with computer code to produce modular routines capable of modelling cake formation, compression, dewatering and washing. The techniques used to describe the operation of the diaphragm filter press are shown and information regarding a typical filter cycle is presented.

\section{FILTER CYCLE DESCRIPTION}

The versatility of the diaphragm filter press design allows the range of operating configurations shown in Figure 1.

The slurry is fed to the chambers of the press through suitable porting with either a centrifugal, diaphragm, single screw or reciprocating pump ${ }^{3}$ such that filtration can occur at constant 
rate/variable pressure or variable rate/variable pressure depending on the type of pump used. If the chambers are not completely filled by cake during this primary filtration stage then a secondary filtration stage may follow where the residual suspension is filtered at constant pressure via movement of the flexible diaphragm. When sufficient suspension has been filtered for the two cakes to meet further cake consolidation via the diaphragm (at constant pressure), cake dewatering by air blowing and/or cake washing can be undertaken in any order to complete the filter cycle prior to cake discharge.

\section{(i) \& (ii) Cake Formation}

Most filtration processes are described by separations technologists through the general filtration equation

$$
\frac{d t_{f}}{d V_{f}}=\frac{\alpha_{a v} c_{c} \mu}{A^{2} \Delta p} V_{f}+\frac{\mu R_{m}}{A \Delta p}
$$

where $\alpha_{a v}$ is the average specific cake resistance and the effective concentration of the solids in the feed $\left(c_{c}\right)$ is given by

$$
c_{c}=\frac{\rho M_{s}}{1-m M_{s}}
$$

In the general sense both the pressure and rate can vary and it is necessary to impose the pump characteristics on the governing equations. Hence equation (1) can be rearranged to

$$
V_{f}=\frac{1}{c_{c} \alpha_{a v}}\left(\frac{A^{2} \Delta p}{\mu Q}-A R_{m}\right)
$$

where $\alpha_{a v}$ is a function of the pressure applied to the solid/liquid mixture through the pumping action. To account for the potential compressibility it is common practice to relate the local specific resistance $(\alpha)$ and porosity $(\varepsilon)$ of filter cakes to the applied pressure over a specified range through constitutive equations such as

$$
\begin{aligned}
& \alpha=\alpha_{0} \Delta p^{n} \\
& \varepsilon=\varepsilon_{0} \Delta p^{n}
\end{aligned}
$$

Combining the integrated form of eqn. (4) (to give an average cake resistance) with eqn. (3) and performing a mass balance on the solids in the cake yields the two equations governing cake formation during the primary filtration phase

$$
\begin{aligned}
& V_{f}=\frac{1}{c_{c} \alpha_{0}(1-n) \Delta p_{c a k e}^{n}}\left(\frac{A^{2} \Delta p}{\mu Q}-A R_{m}\right) \\
& h=2\left(\frac{V_{f} M_{s}\left(\frac{\rho_{s}}{\rho}(m-1)+1\right)}{A \frac{\rho_{s}}{\rho}\left(1-m M_{s}\right)}\right)
\end{aligned}
$$


When unfiltered suspension remains in the filter chamber(s) a secondary filtration phase may follow the initial primary filtration. If the secondary phase is required then further filtration at constant pressure is initiated via the diaphragm, with filtrate draining through one filtration surface in each chamber. Modifying eqn. (1) to account for the effective increase in resistance to filtration due to the presence of a filter cake on the filter medium at the start of the filtration gives the governing equation

$$
\frac{t_{f}-t_{t r}}{V_{f}-V_{t r}}=\frac{\alpha_{a v} c_{c} \mu}{(A / 2)^{2} \Delta p}\left(V_{f}+V_{t r}\right)+\frac{\mu}{(A / 2) \Delta p}\left(\frac{\alpha_{a v} c_{p} V_{p}}{(A / 2)}+R_{m}\right)
$$

Thus, if the pump characteristics relating $\Delta p$ and $Q$ and the constitutive equations relating $\alpha$ and $\varepsilon$ (and hence $m$ ) to $\Delta p$ are known from laboratory scale tests, cake formation can be modelled.

\section{(iii) Cake Consolidation}

The cake consolidation is performed at constant pressure through use of the filter diaphragm and is analysed via an empirical relationship relating the consolidation ratio

$$
U_{c}=\frac{h_{o}-h}{h_{o}-h_{\infty}}
$$

to a dimensionless consolidation time defined by

$$
T_{c}=\frac{\left(n_{1}\right)^{2} C_{c} t_{c}}{\omega_{0}^{2}}
$$

such that

$$
U_{c}=\frac{\sqrt{4 T_{c} / \pi}}{\left(1+\left(4 T_{c} / \pi\right)^{v}\right)^{1 / 2 v}}
$$

where $C_{c}$ is the consolidation coefficient and $v$ the consolidation behaviour index. Values for $v$ and the other scale-up constants in the constitutive equations for the consolidation phase can be obtained through laboratory tests with, for instance, a piston press (as previously described ${ }^{4}$ ) and related through eqns. (9)-(11) to give expressions for cake height and filtrate volume during the consolidation period(s).

Consolidation is assumed to end when the flow rate of liquid from the filter falls below a predetermined level.

\section{(iv) Cake Dewatering (by air blowing)}

The dewatering phase is analysed through a combination of empirical and theoretical relations. These facilitate the calculation of the time required to achieve a given filter cake moisture content and the air flow rate through the cake during the dewatering period and have been described in detail previously ${ }^{5-7}$. Two parameters of prime importance in dewatering are the pressure required to initiate dewatering $\left(p_{b}\right)$ and the irreducible saturation of the filter cake under consideration $\left(S_{\infty}\right)$. Although these should be measured experimentally, the former can be determined with sufficient accuracy from 
$p_{b}=\frac{4.6\left(1-\varepsilon_{a v}\right) \sigma}{\varepsilon_{a v} x_{a v}}$

and correlations exist (for relatively large, granular materials) which allow the estimation of $S_{\infty}$

$$
\begin{aligned}
& N_{c a p}=\frac{\varepsilon_{a v}^{3} x_{a v}^{2}\left(\rho g h+\Delta p_{d}\right)}{\left(1-\varepsilon_{a v}\right)^{2} h \sigma} \\
& S_{\infty}=0.155\left(1+0.031 N_{c a p}^{-0.49}\right)
\end{aligned}
$$

With data for $p_{b}$ and $S_{\infty}$ available, and assuming that (i) no dewatering of the cake occurs through evaporation of the mother liquor, and (ii) the displacing fluid is saturated instantly as it enters the cake, a dimensionless saturation $\left(S_{R}\right.$, termed the reduced saturation) can be related empirically to a dimensionless time $(\theta)$

$$
\begin{aligned}
& S_{R}=\frac{S-S_{\infty}}{1-S_{\infty}} \\
& \theta=\left(\frac{1}{b_{2}}\left(\frac{1}{S_{R}}-1\right)\right)^{1 / b_{3}}
\end{aligned}
$$

where $b_{2}$ and $b_{3}$ are known empirical constants. The dewatering time $\left(t_{d}\right)$ is then estimated from

$$
t_{d}=\frac{\theta \varepsilon_{a v} \mu h^{2}\left(1-S_{\infty}\right)}{\Delta p_{d} k_{a v}}
$$

Allowing for losses in the feed pipes to the filter and the effects of plant elevation, the air flow rate required to achieve the desired moisture content can be calculated ${ }^{6,7}$.

\section{(v) Cake Washing (by displacement)}

The technique used in the analysis of the washing phase(s) employs the dispersion model ${ }^{7-10}$. Use of the model requires a superficial wash velocity

$$
u_{o}=\frac{\Delta p_{w}}{\mu_{w}\left(\alpha_{a v} \rho_{s} h\left(1-\varepsilon_{a v}\right)+R_{m}\right)}
$$

and the product Re.Sc to be calculated

$\operatorname{Re} . \mathrm{Sc}=\frac{u_{o} x_{a v}}{\varepsilon_{a v} D}$

The ratio $D_{L} / D$ can then be evaluated according to the filter cake height

$$
\frac{D_{L}}{D}=0.707+55(\operatorname{Re} . \mathrm{Sc})^{0.96} 0 \leq h \leq 0.1 \mathrm{~m}
$$


$\frac{D_{L}}{D}=0.707+1.75($ Re.Sc $) \quad h>0.1 \mathrm{~m}$

and the dispersion number is then given by

$D_{n}=\frac{(\operatorname{Re} . S c) h}{x_{a v}\left(D_{L} / D\right)}$

With a knowledge of the number of wash ratios to be applied the washing time $\left(t_{w}\right)$, effluent solute concentration $(c)$ and fractional solute recovery $(F)$ can be estimated from known, experimentally proven, correlations when allowances for cake saturation and scale are included ${ }^{7}$.

\section{AN EXAMPLE OF FILTER SIMULATION}

The above methodologies enable flexible simulations to be developed. When suitably applied these can model most combinations of cake formation, consolidation, dewatering and washing to allow preliminary sizing and scale-up of equipment to be performed. Although the example of a diaphragm press has been chosen, the procedures can be readily adapted to model the performance of other filtration devices such as tube filter presses and rotary vacuum filters. The data shown below illustrate how information associated with the entire filter cycle can be produced for a set of typical operating conditions through software simulation techniques.

\section{Example Problem}

A diaphragm filter press has been identified as being potentially suited to the task of producing a nominal 5 te of dry particulate solids from an $8 \%$ w/w slurry. Following the formation stage(s), the homogeneity of the cake is to be improved through consolidation with the diaphragm. The resultant compact is then to be dewatered by air blowing until a moisture content of $20 \%$ is achieved and subsequently washed until the solute concentration in the cake is reduced from 30 to $2.5 \mathrm{~kg} \mathrm{~m}^{-3}$. The feed is delivered to the filter press via a centrifugal pump (see Table 1 for the characteristic curve of the pump) and the feed suspension characteristics have been experimentally determined (see Table 2 ).

Determine if the separation can be achieved, the required filtration time, the filter area and other performance indicators.

\section{Solution via Simulation}

When the computer simulation package is used to model the above problem a wealth of data related to both the individual phases and the total filter cycle is produced. Figures 2-4 give examples. The data suggest that the required objectives of the separation can be readily achieved with the diaphragm press and pump specified. The filter area and total cycle time required are estimated to be $380.3 \mathrm{~m}^{2}$ and $7678 \mathrm{~s}$ respectively with the filtration, consolidation, dewatering and washing phases projected to end at times corresponding to $2028 \mathrm{~s}, 4056 \mathrm{~s}, 4707 \mathrm{~s}$ and $7678 \mathrm{~s}$.

During the period of the primary filtration the cakes in each of the filter chambers progressively grow to a combined height (noting that cake formation occurs on two surfaces in each chamber) of $34 \mathrm{~mm}$ whilst the pressure in the chambers increases from 49.7 to $94 \mathrm{kPa}$ as the cakes form. At the end of the primary filtration period $\left(t_{f}=1138 \mathrm{~s}\right)$ un-filtered suspension still remains in the chambers and a secondary filtration at constant pressure is required. Within this period the cakes in each chamber continue to form and eventually join to achieve the maximum allowable height of $40 \mathrm{~mm}$ (i.e. $2 \times 20 \mathrm{~mm}$ ) after $2028 \mathrm{~s}$. At the end of the cake formation stages the chamber and cake 
moisture contents are identical and equal to $52.1 \%$ with the total solids, liquid and solute masses in the filter estimated at 5092, 5534 and $166 \mathrm{~kg}$ respectively.

Following the cake formation phases the consolidation, dewatering and washing phases are sequentially initiated. During the period of consolidation the cake moisture and solute masses are reduced to $27 \%$ and $57 \mathrm{~kg}$ respectively whilst the cake height is also reduced to $21 \mathrm{~mm}$ and is assumed to remain at that value for the subsequent dewatering and washing phases. The solute mass and moisture content of the cake are further lowered to $38 \mathrm{~kg}$ and the desired $20 \%$ respectively at the end of the dewatering period using an overall air rate of $1.8 \times 10^{-5} \mathrm{~m}^{3} \mathrm{~m}^{-2} \mathrm{~s}^{-1}$. In the washing period, 3.5 wash ratios (based on the unsaturated cake) are used to produce the required solute concentration of $2.5 \mathrm{~kg} \mathrm{~m}^{-3}$; the fractional recovery for the washing period alone being $88 \%$.

At the end of the filter cycle it is estimated that $5092 \mathrm{~kg}$ of solids, $1891 \mathrm{~kg}$ of liquid and $4.7 \mathrm{~kg}$ of solute remain in the filter press. The fractional recovery of solute for the whole cycle is calculated to be $97.2 \%$.

\section{CONCLUDING REMARKS}

The above considerations give an indication of the manner in which computer simulations can significantly improve the understanding of the operations which can occur during the filter cycle. Each of the theories used to model the cake formation, consolidation, dewatering and washing phases has been proven in pilot scale and industrial tests. It is apparent that the modelling procedures adopted for the simulation of the diaphragm filter press can be readily adapted to include optimisation routines and extended to encompass other types of separation equipment. When fully developed, such computer software should provide a suite of programs which are sufficiently powerful to minimise the number of costly and time consuming experiments which need to be performed to assess the suitability of equipment for a particular duty.

\section{NOMENCLATURE}

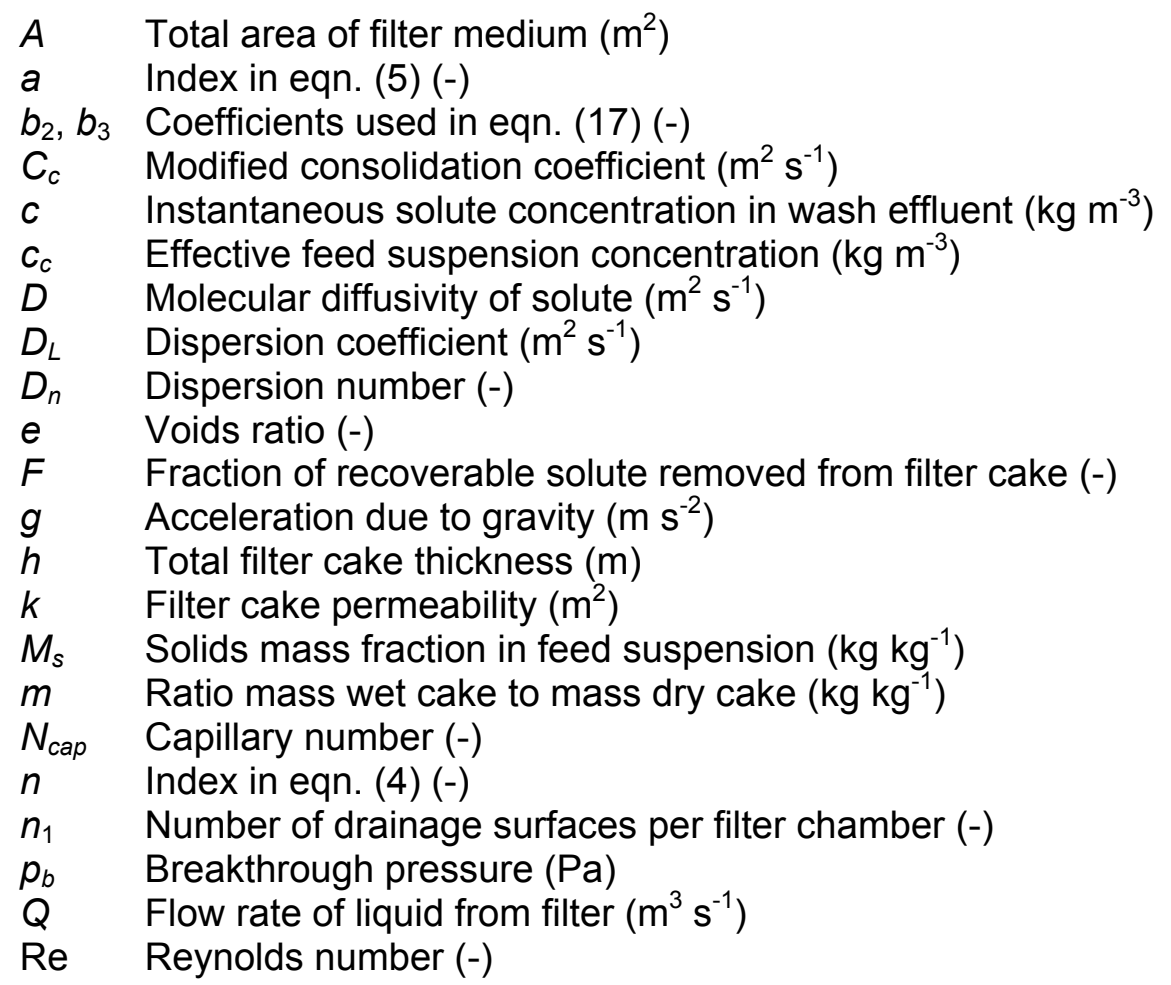


$R_{m} \quad$ Filter medium resistance $\left(\mathrm{m}^{-1}\right)$

$S \quad$ Filter cake saturation (-)

$S_{R} \quad$ Reduced saturation (-)

$S_{\infty} \quad$ Irreducible saturation (-)

Sc Schmidt number (-)

$T_{c} \quad$ Dimensionless consolidation time (-)

$t \quad$ Time (s)

$U \quad$ Consolidation ratio (-)

$u_{0} \quad$ Superficial wash velocity $\left(\mathrm{m} \mathrm{s}^{-1}\right)$

$V \quad$ Cumulative volume of liquid produced from filter $\left(\mathrm{m}^{3}\right)$

$x_{a v} \quad$ Mean particle size in feed suspension (m)

Greek symbols

$\alpha \quad$ Local specific cake resistance $\left(\mathrm{m} \mathrm{kg}^{-1}\right)$

$\alpha_{a v} \quad$ Average specific cake resistance $\left(\mathrm{m} \mathrm{kg}^{-1}\right)$

$\alpha_{0} \quad$ Specific cake resistance at $\Delta p=1 \mathrm{kPa}\left(\mathrm{m} \mathrm{kg}^{-1}\right)$

$\Delta p \quad$ Pressure applied to solid/liquid mixture ( $\mathrm{Pa}$ or $\mathrm{kPa})$

$\Delta p_{\text {cake }}$ Pressure drop over filter cake $(\mathrm{Pa})$

$\varepsilon \quad$ Local filter cake porosity (-)

$\varepsilon_{a v} \quad$ Average filter cake porosity (-)

$\varepsilon_{0} \quad$ Filter cake porosity at $\Delta p=1 \mathrm{kPa}(-)$

$\theta \quad$ Dimensionless time (-)

$\mu \quad$ Liquid viscosity (Pa s)

$v \quad$ Consolidation behaviour index (-)

$\rho \quad$ Liquid density $\left(\mathrm{kg} \mathrm{m}^{-3}\right)$

$\rho_{s} \quad$ Solids density $\left(\mathrm{kg} \mathrm{m}^{-3}\right)$

$\sigma \quad$ Liquid surface tension $\left(\mathrm{N} \mathrm{m}^{-1}\right)$

$\omega_{0} \quad$ Volume of solids produced per unit area of drainage surface $\left(\mathrm{m}^{3} \mathrm{~m}^{-2}\right)$

Subscripts

c consolidation phase

d dewatering phase

$f \quad$ filtration phase

o start of phase

$p \quad$ end of previous phase

tr transition from primary to secondary filtration phase

w washing phase

$\infty \quad$ at equilibrium

\section{REFERENCES}

1. E.S. Tarleton and R.J. Wakeman, Solid/Liquid Separation Equipment Simulation \& Design: $p^{C}$ SELECT - Personal computer software for the analysis of filtration and sedimentation test data and the selection of solid/liquid separation equipment, Separations Technology Associates, 1991.

2. R.J. Wakeman and E.S. Tarleton, Solid/liquid separation equipment simulation and design - an expert systems approach, Filtration and Separation, 28(4), 268-274, 1991.

3. I.M. Young, in Solid/liquid Separation Equipment Scale-up, $2^{\text {nd }}$ Ed., D.B. Purchas and R.J. Wakeman (Eds.), pp.446-484, Uplands Press and Filtration Specialists, London, 1986.

4. R.J. Wakeman, M.N. Sabri and E.S. Tarleton, Factors affecting the formation and properties of wet compacts, Powder Technology, 65(1-3), 283-292, 1991. 
5. R.J. Wakeman, Vacuum dewatering and residual saturation of incompressible filter cakes, International J. Mineral Processing, 3, 193-206, 1976.

6. R.J. Wakeman, An improved analysis for the forced gas deliquoring of filter cakes and porous media, J. Separation Process Technology, 3, 32-38, 1982.

7. R.J. Wakeman and E.S. Tarleton, Modelling, simulation and process design of the filter cycle, Filtration and Separation, 27(6), 412-419, 1990.

8. R.J. Wakeman, Transport equations for filter cake washing, Chemical Engineering Research and Design, 64, 308-319, 1986.

9. R.J. Wakeman and G.J. Attwood, Developments in the application of cake washing theory, Filtration and Separation, 25, 272-275, 1988.

10. R.J. Wakeman and G.J. Attwood, Simulations of dispersion phenomena in filter cake washing, Chemical Engineering Research and Design, 68, 161-171, 1990. 
FIGURES AND TABLES

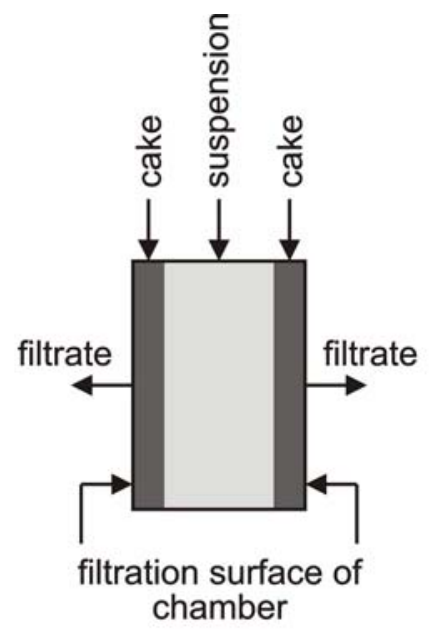

(i) Primary filtration

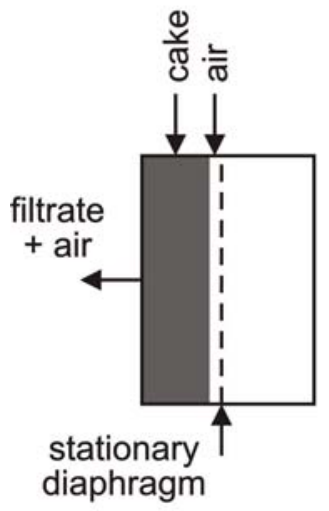

(vi) Dewatering

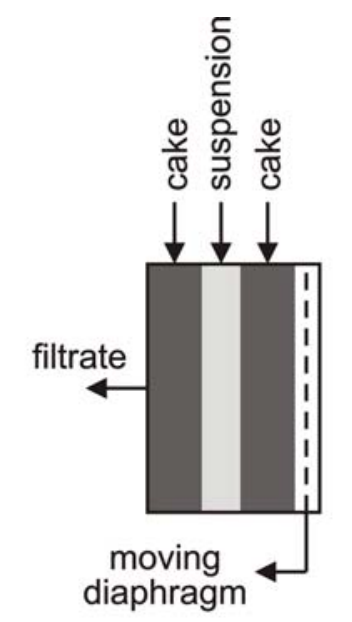

(ii) Secondary filtration

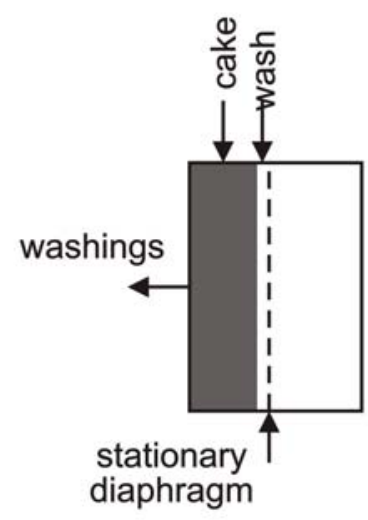

(v) Washing

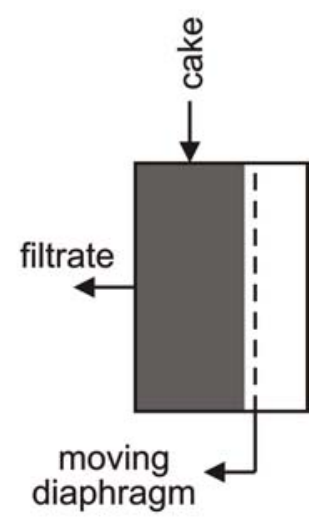

(iii) Consolidation

Figure 1: Possible operations in a diaphragm filter press. 


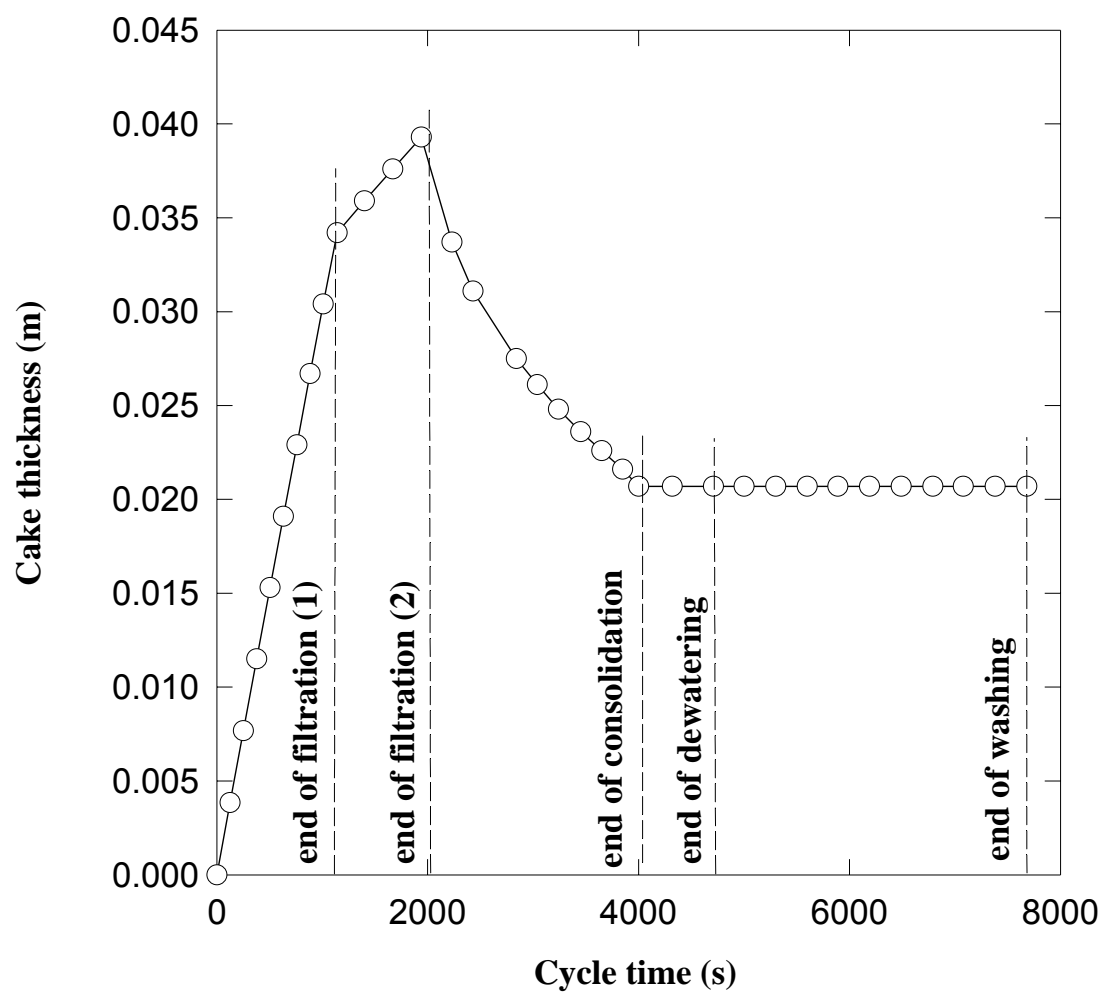

Figure 2: Total cake thickness per chamber vs. filter cycle time.

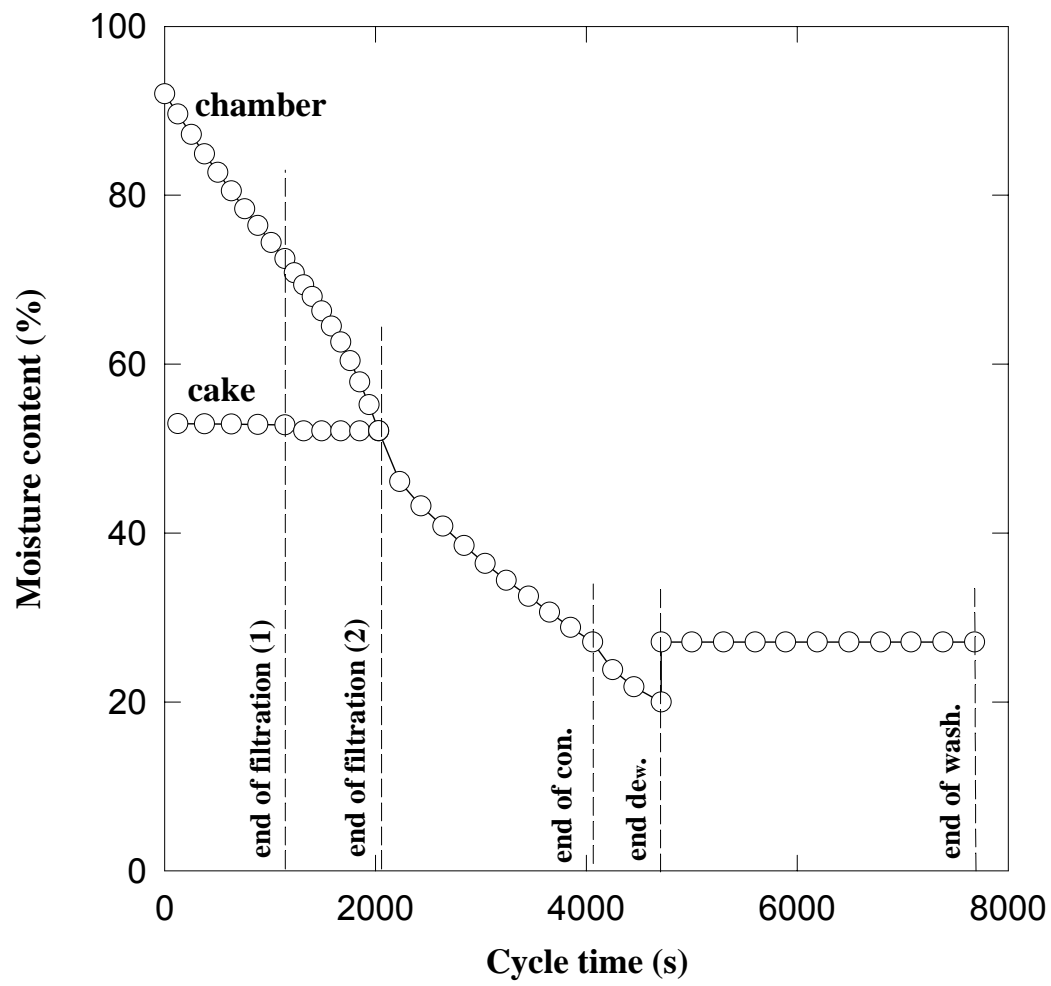

Figure 3: Chamber and cake moisture contents vs. filter cycle time. 


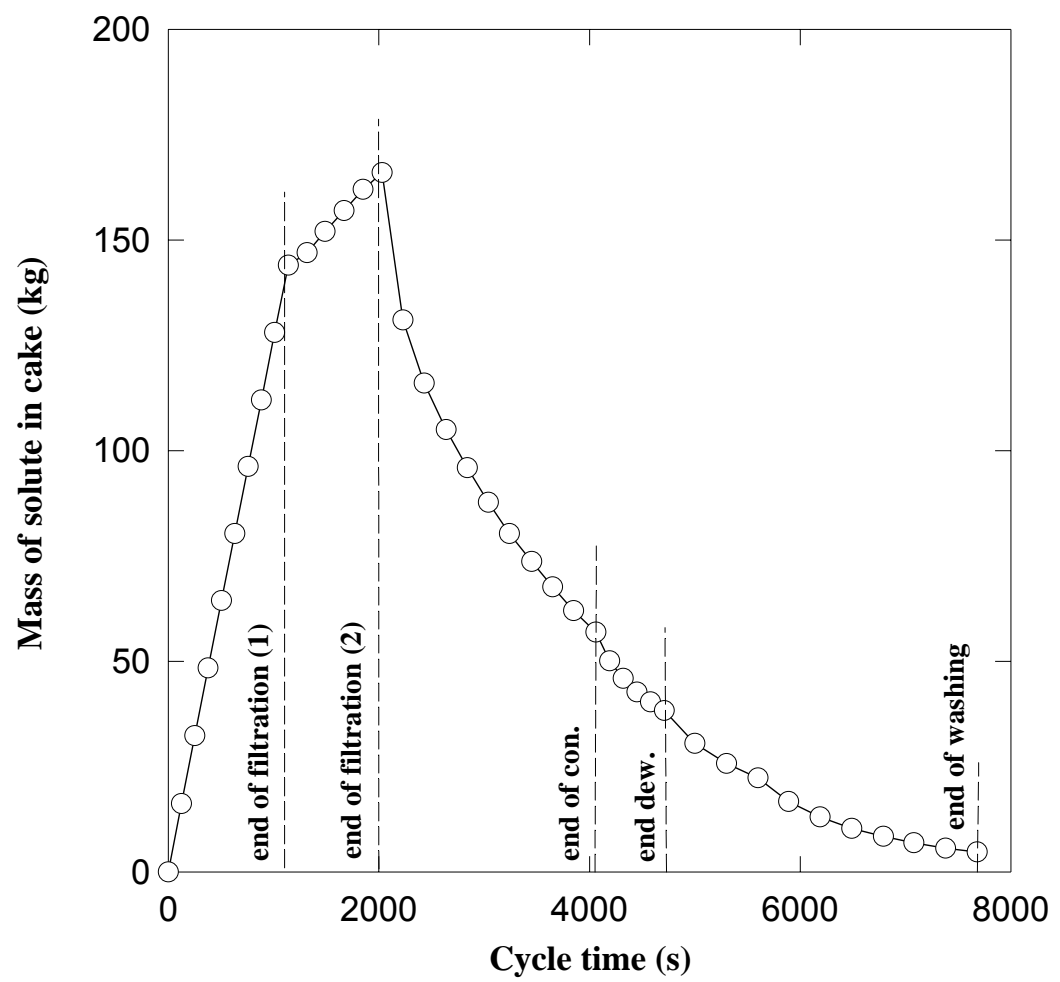

Figure 4: Mass of solute in cake liquor vs. filter cycle time.

\begin{tabular}{|c|c|}
\hline Flow rate $\left(\mathrm{m}^{3} \mathrm{~s}^{-1}\right)$ & Pressure $(\mathrm{kPa})$ \\
\hline 0 & 360 \\
0.022 & 300 \\
0.03 & 250 \\
0.035 & 200 \\
0.037 & 150 \\
0.0385 & 100 \\
0.0395 & 50 \\
0.04 & 0 \\
\hline
\end{tabular}

Table 1: Pump characteristics. 


\begin{tabular}{|l|l|}
\hline Filter chamber thickness & $80 \mathrm{~mm}$ \\
Filter medium resistance & $3 \times 10^{11} \mathrm{~m}^{-1}$ \\
Max. cake thick. on each filter surface & $20 \mathrm{~mm}$ \\
Density of solids & $2500 \mathrm{~kg} \mathrm{~m}^{-3}$ \\
Density of filtrate and wash & $998 \mathrm{~kg} \mathrm{~m}^{-3}$ \\
Viscosity of filtrate and wash & $0.001 \mathrm{~Pa} \mathrm{~s}$ \\
Surface tension of filtrate and wash & $0.07 \mathrm{~N} \mathrm{~m}{ }^{-1}$ \\
Solids concentration in feed & $8 \% \mathrm{~W} / \mathrm{w}$ \\
Mean size of solids in feed & $10 \mu \mathrm{m}$ \\
Secondary filtration pressure (if needed) & $600 \mathrm{kPa}$ \\
Consolidation index & 3 \\
Consolidation and washing pressure & $600 \mathrm{kPa}$ \\
Dewatering pressure & $400 \mathrm{kPa}$ \\
Barometric pressure & $100 \mathrm{kPa}$ \\
Irreducible cake saturation & 0.26 \\
Viscosity of air & $1.8 \times 10^{-5} \mathrm{~Pa} \mathrm{~s}$ \\
Solute concentration in feed & $30 \mathrm{~kg} \mathrm{~m}^{-3}$ \\
Solute concentration in wash & $0.5 \mathrm{~kg} \mathrm{~m}^{-3}$ \\
Solute diffusivity & $1 \times 10^{9} \mathrm{~m}^{2} \mathrm{~s}^{-1}$ \\
Constitutive eqns. for filtration & $\alpha=6 \times 10^{9} \Delta p^{0.6} ; e=3-0.1 \log (\Delta p)$ \\
Constitutive eqns. for consolidation & $e=1.63-0.55 \log (\Delta p) ; C_{c}=1 \times 10^{-8} \Delta p^{0.1}$ \\
\hline
\end{tabular}

Table 2: Values used in simulation. 